\title{
Time to Peak Concentration of Amikacin in the Antebrachiocarpal Joint Following Cephalic Intravenous Regional Limb Perfusion in Standing Horses
}

\author{
Kajsa Gustafsson ${ }^{1}$ Amos J. Tatz ${ }^{1}$ Roee Dahan ${ }^{1}$ Malka Britzi ${ }^{2}$ Stefan Soback ${ }^{2}$ Gila A. Sutton ${ }^{1}$ \\ Gal Kelmer ${ }^{1}$ \\ ${ }^{1}$ Department of Large Animal Medicine and Surgery, Koret School of \\ Address for correspondence Kajsa Gustafsson, DVM, Department of \\ Veterinary Medicine, The Robert H. Smith Faculty of Agriculture, \\ Large Animal Medicine and Surgery, Koret School of Veterinary \\ Medicine, The Robert H. Smith Faculty of Agriculture, Food and \\ Food and Environment, the Hebrew University of Jerusalem, \\ Rehovot, Israel \\ 2 National Residue Control Laboratory, Kimron Veterinary Institute, \\ Beit Dagan, Israel \\ Environment, the Hebrew University of Jerusalem, Rehovot, Israel \\ (e-mail: kajsagustafsson1@hotmail.com).
}

Vet Comp Orthop Traumatol 2020;33:327-332.

\begin{abstract}
Keywords

- regional limb perfusion

- horse

- amikacin sulphate

- radiocarpal joint

- tourniquet

Objective The aim of this study was to determine the time $\left(T_{\max }\right)$ to the maximum concentration $\left(C_{\max }\right)$ of amikacin sulphate in synovial fluid of the radiocarpal joint $(R C J)$ following cephalic intravenous regional limb perfusion (IVRLP) using $2 \mathrm{~g}$ of amikacin sulphate. Methods Cephalic IVRLP was performed with $2 \mathrm{~g}$ of amikacin sulphate diluted in $0.9 \%$ $\mathrm{NaCl}$ to a total volume of $100 \mathrm{~mL}$ in six healthy adult mixed breed mares. An Esmarch's rubber tourniquet was applied for 30 minutes and the antibiotic solution was infused through a 23-gauge butterfly catheter. Synovial fluid was collected from the RC] prior to the infusion and at 5, 10,15,20,25 and 30 minutes after completion of IVRLP. The tourniquet was removed after the last arthrocentesis. Synovial fluid amikacin sulphate concentrations were determined by liquid chromatography/tandem mass spectrometry.

Results The calculated mean $\mathrm{T}_{\max }$ occurred at 15 minutes (range: 10-20 minutes) post-perfusion. The highest synovial fluid amikacin sulphate concentration was noted at 10 minutes in 2 horses, 15 minutes in 2 horses and 20 minutes in 2 horses. The highest mean concentration was $1023 \mu \mathrm{g} / \mathrm{mL}$ and was noted at 20 minutes. Synovial mean concentrations were significantly different between 15 and 30 minutes.

Clinical Significance In this study no $T_{\max }$ occurred after 20 minutes; thus, 30 minutes of tourniquet application time appear to be excessive. The 20 minutes duration of tourniquet application appears sufficient for the treatment of the RC] in cephalic IVRLP using $2 \mathrm{~g}$ amikacin sulphate in a total volume of $100 \mathrm{~mL}$.
\end{abstract}

\section{Introduction}

Intravenous regional limb perfusion (IVRLP) is an effective technique for delivering high concentrations of antimicrobial agents to synovial structures of the distal limb in horses suffering from septic arthritis or osteomyelitis. ${ }^{1}$ Septic arthritis and osteomyelitis are challenging to treat and require immediate aggressive treatment to save the career and even the life of affected horses. ${ }^{2,3}$ Therapeutically effective antimicrobial tissue and synovial fluid concentrations in the distal limb are difficult to reach through systemic routes of administration. ${ }^{4}$ Also, administration of systemic antimicrobials may result in potentially fatal side effects such as antibiotic-induced colitis, which can be avoided through local administration. ${ }^{5}$

Amikacin sulphate is the most commonly used antimicrobial in IVRLP in both clinical and experimental received

November 17, 2019

accepted after revision

May 12, 2020

published online

August 16, 2020 (c) 2020 Georg Thieme Verlag KG Stuttgart · New York
DOI https://doi.org/ $10.1055 / \mathrm{s}-0040-1714418$. ISSN 0932-0814. 
settings. ${ }^{6-16}$ Amikacin sulphate is a concentration-dependent antibiotic medication of the aminoglycoside class of antimicrobials that is active against most common aerobic pathogens isolated from equine orthopaedic infections. ${ }^{17}$ It is the most common choice of antimicrobial in cases of septic arthritis or osteomyelitis prior to obtaining culture and sensitivity results. ${ }^{18}$ Additionally, the concentration-dependent pharmacological activity of amikacin sulphate, including its a long post-antibiotic effect, makes it a suitable candidate for delivery of regional high concentrations. ${ }^{19}$ The bactericidal effect and extent of post-antibiotic effect of amikacin sulphate are dependent on the maximum concentration:minimum inhibitory concentration ratio $\left(\mathrm{C}_{\max }: \mathrm{MIC}\right)$ achieved in synovial fluid after administration. ${ }^{19}$ A $C_{\max }$ :MIC ratio of 8:1-10:1 has been suggested for effective treatment of orthopaedic infections with amikacin sulphate in horses. ${ }^{13,20}$

Administration of amikacin sulphate through IVRLP avoids nephrotoxic effects associated with prolonged systemic administration of aminoglycosides in horses, with expected low serum concentrations also after the release of the tourniquet. ${ }^{16}$ On the contrary to described positive aspects of a local administration, a recent study concluded that intra-articular administration with as low as $250 \mathrm{mg}$ amikacin sulphate was markedly cytotoxic to joint cells. ${ }^{21}$ Adverse effects of high amikacin sulphate concentrations to joint cells delivered through IVRLP have not been investigated.

Intravenous regional limb perfusion in standing horses requires heavy sedation due to pain and discomfort caused by the tourniquet. ${ }^{22}$ Post-sedation effects are a concern in continuous treatment when daily heavy sedation is required for an extended period, causing delayed gastric emptying which could lead to colic. ${ }^{23,24}$ Additionally, the use of tourniquets in human medicine has been associated with ischaemiareperfusion injuries, neural ischaemia and wound hypoxia, leading to a recommended tourniquet application time of less than 1 hour. ${ }^{25,26}$ In cattle, side effects due to reperfusion injury have been observed after 90 minutes of tourniquet application time. ${ }^{27}$ These adverse effects, although not investigated, could also be present in the equine population advocating for the tourniquet application time to be as short as possible. The current recommended tourniquet application time for standing horses in regional limb perfusion is 30 minutes in clinical settings and in experimental studies. ${ }^{1,28}$

Several studies have investigated the ideal tourniquet application time for IVRLP by comparing synovial fluid concentrations at different time points post-infusion. Previously it has been suggested that the time to peak concentration $\left(\mathrm{T}_{\max }\right)$ occurs 15 minutes post-infusion after IVRLP with $1.25 \mathrm{~g}$ of amikacin sulphate in the lateral/medial palmar/plantar vein of horses under general anaesthesia. ${ }^{9} \mathrm{~A}$ recent report concluded that 20 minutes of tourniquet application time resulted in similar synovial fluid antimicrobial concentrations in both the radiocarpal joint and metacarpophalangeal joint as did a 30 minute application time, when $1 \mathrm{~g}$ of amikacin sulphate was infused in cephalic IVRLP utilizing a pneumatic tourniquet. ${ }^{10}$ The same group found no significant difference in synovial fluid concentrations of amikacin sulphate in the radiocarpal joint and metacarpophalangeal joint following a
10 and a 30 minutes tourniquet application time. ${ }^{29}$ In these studies, the joints were evaluated separately, only two time points were compared and the synovial fluid amikacin sulphate concentration throughout the complete tourniquet application time was not evaluated.

Based on a current literature search, there are no studies investigating the appropriate tourniquet application time for cephalic IVRLP by synovial fluid sampling of the radiocarpal joint in 5 minutes intervals. Such a model is needed to collect more data and enable comparison between several time points throughout the first 30 minutes of tourniquet application time. The aim of this study was to establish the time to peak concentration $\left(\mathrm{T}_{\max }\right)$ of amikacin sulphate in synovial fluid of the radiocarpal joint after cephalic IVRLP with $2 \mathrm{~g}$ of amikacin sulphate in standing, sedated horses. Our hypothesis was that the $\mathrm{T}_{\max }$ would occur before the 30 -minute time point in all horses.

\section{Materials and Methods}

\section{Animals}

Six $(n=6)$ adult mixed breed mares were used in the study. The ages of the participating horses ranged from 2 to 18 years (median: 9.5 years) and their body weight ranged from 285 to $470 \mathrm{~kg}$ (median: $372 \mathrm{~kg}$ ). Prior to commencement of the study, all horses underwent thorough physical examinations and their haematology were assessed. Only horses with no observed lameness at a walk, no evidence of orthopaedic problems on palpation and normal clinical and haematological values were included. All horses were given tetanus and influenza vaccinations 2 weeks prior to the initiation of the study and were housed individually with hay and water $a d$ libitum. The study was approved by the University Animal Care and Use Committee (MD-16-14812-3).

\section{Regional Limb Perfusion and Sampling}

One forelimb of each horse $(n=6)$ was randomly assigned to treatment (by coin toss). The area of the medial, palmar and dorsal radius was aseptically prepared and local peri-neural anaesthesia was performed with mepivacaine $\mathrm{HCl} 2 \%$ (Mepivacaine, Ceva Animal Health, Glenorie, Australia), to desensitize the ulnar, median and musculocutaneous nerves, with a volume of $10 \mathrm{~mL}$ at each location. ${ }^{30} \mathrm{~A} 10 \times 5 \mathrm{~cm}$ area overlying the cephalic vein at the level of the chestnut was clipped and aseptically prepared. All horses received IV detomidine hydrochloride (Domosedan, Orion Pharma Animal Health, Espoo, Finland) $0.01 \mathrm{mg} / \mathrm{kg}$ and IV butorphanol tartrate (Butomidor, Richter Pharma AG, Wels, Austria) $0.01 \mathrm{mg} / \mathrm{kg}$, one time through IV injection, prior to tourniquet application.

Two $10 \mathrm{~cm}$ rolled non-elastic gauze bandages (Sion Medical, Sderot, Israel) were placed over the vasculature proximal to the prepared infusion site in the cephalic vein and an $8 \mathrm{~cm}$ wide rubber Esmarch's tourniquet (Degania Medical, Degania Bet, Israel) was placed on top of the gauze with the distal border of the tourniquet $5 \mathrm{~cm}$ proximal to the chestnut. A 23 gauge and $2 \mathrm{~cm}$ long butterfly catheter was inserted in an aseptic manner in the cephalic vein and directed distally. After blood flow into the extension set was observed, the catheter 
was secured to the skin with cyanoacrylate adhesive. Subsequently, the catheter was used for infusion of $4 \mathrm{~mL}(2 \mathrm{~g})$ of amikacin sulphate (Amikacin, Anfarm Hellas, Attiki, Greece), diluted in $96 \mathrm{~mL}$ of $0.9 \% \mathrm{NaCl}$ to a total volume of $100 \mathrm{~mL} .{ }^{16}$ The solution was manually infused continuously over $\sim 2$ minutes, using five $20 \mathrm{~mL}$ syringes.

The dorsal aspect of the carpus was clipped, and aseptically prepared one time. Synovial fluid $(1 \mathrm{~mL})$ was collected percutaneously from the radiocarpal joint using a 21 -gauge, $4 \mathrm{~cm}$ long needle between the distal radius and the proximal articular surface of the radiocarpal bone, lateral to the extensor carpi radialis tendon while lifting the forelimb in a slightly flexed position. Samples were collected in empty urine collection tubes (BD Vacutainer, New Jersey, United States) before infusion (time 0 ) and at 5, 10, 15, 20, 25 and 30 minutes post perfusion. The tourniquet was kept in place during the synovial sampling and removed 30 minutes after termination of the antimicrobial infusion. Samples were centrifuged and the supernatant frozen at $-80^{\circ} \mathrm{C}$ in empty $2 \mathrm{~mL}$ plastic vials until processed for amikacin sulphate concentration determination 8 weeks after the samples were obtained.

After sampling was completed, all horses received phenylbutazone at $2.2 \mathrm{mg} / \mathrm{kg}$ IV twice daily for 2 days. They were walked and evaluated daily for 7 days for lameness or other complications related to venipuncture and arthrocentesis.

\section{Synovial Fluid Preparation}

Samples were prepared and analyzed using liquid chromatography/tandem mass-spectrometry as previously described. ${ }^{16}$ Briefly, synovial fluid samples were mixed with double-distilled water, followed by acetonitrile for protein precipitation. The extract was mixed with hexane and dichloromethane and finally, the aqueous phase was analysed by liquid chromatography/tandem mass-spectrometry. Agilent 1200 (Agilent Technologies, Waldbronn, Germany) liquid chromatography system (binary pump, degasser, heated column compartment and autosampler) connected to Applied Biosystems API 4000 (Applied Biosystems, Toronto, ON, Canada) mass spectrometer was utilized. The chromatographic separation was performed on a Symmetry C18 column ( $3.5 \mu$, $2.1 \times 100 \mathrm{~mm}$, Waters, Massachusetts, United States)

Calibration curves were prepared by adding known amounts of amikacin sulphate to drug-free synovial fluid within the range of 0.5 to $2500 \mu \mathrm{g} / \mathrm{mL}$.

Each calibration curve was prepared on three occasions, in triplicate on three different days. The accuracy, intra-day and inter-day precision were calculated for the lower level of quantification, the mid-level of quantification and the high level of quantification $(0.5,250,2,500 \mu \mathrm{g} / \mathrm{mL})$.

\section{Statistical Methods}

$\mathrm{C}_{\max }$ and $\mathrm{T}_{\max }$ were determined by visual observation of the data. The sphericity assumption of the data was assessed using Mauchly test of sphericity. Repeated measure analysis of variance (ANOVA) test was used to assess the effects of time on synovial fluid amikacin sulphate concentrations. The correlation between body weight and synovial fluid amikacin sulphate concentration was assessed using Spearman correlation. Post-hoc analysis was performed through repeated measures ANOVA pair wise comparisons default (Least Significant Difference test). Statistical analyses were performed using statistical software program SPSS (IBM SPSS STATISTICS software version 25, IBM, New York, United States). Statistical significance was set at $p<0.05$.

\section{Results}

All baseline synovial fluid amikacin sulphate concentrations were $0 \mu \mathrm{g} / \mathrm{mL}$. The mean synovial fluid amikacin sulphate $\mathrm{T}_{\max }$ was 15 minutes (range: 10-20 minutes) and the mean highest concentration $\left(C_{\max }\right)$ was $1153 \mu \mathrm{g} / \mathrm{mL}$ (range: 588-1950 $\mu \mathrm{g} / \mathrm{mL}$ ) (-Table 1). There was no significant correlation between synovial fluid amikacin sulphate concentration and body weight $(\mathrm{Rho}=0.143, p=0.79)$.

The mean synovial fluid amikacin sulphate concentration increased until 20 minutes and, subsequently, decreased until 30 minutes which was the last sampling point (-Fig. 1). The overall change in mean amikacin sulphate concentration was significant over time. In post-hoc analysis mean amikacin sulphate synovial fluid concentrations at all time points were significantly different to Time 0 and to the 5 -minute time point. For the remaining time points, significant differences were found in the mean amikacin sulphate synovial fluid concentration only when comparing the concentration at 15 minutes to 30 minutes (-Fig. $\mathbf{1}$ ).

No significant arthrocentesis or venipuncture related adverse effects were observed throughout the study. Two horses had mild visible swelling at the site of infusion throughout the IVRLP and were suspected to have minor perivascular administration of the perfusate. All samples were obtained and analysed successfully. Accuracy of the synovial fluid analysis was within the range of 92 to $107 \%$ and the precision was within the range of 7.6 to $14.3 \%$.

\section{Discussion}

The time of peak concentration of amikacin sulphate in the synovial fluid of the radiocarpal joint, following IVRLP in standing horses, was observed between 10 and 20 minutes from the completion of the amikacin sulphate infusion. This supports our hypothesis that $\mathrm{T}_{\max }$ of amikacin sulphate in synovial fluid would occur before the 30 -minute time point when delivered through cephalic IVRLP in the current formulation. The findings of this study were in agreement with the recent reports suggesting that 20 minutes of tourniquet application time for IVRLP are sufficient to reach $\mathrm{T}_{\max }$ of amikacin sulphate in the distal interphalangeal and metacarpophalangeal joints. ${ }^{9,10,31}$ Thus, the widely used and reported 30-minute tourniquet application time appears excessive, when measuring concentrations of amikacin sulphate in the radiocarpal joint and other joints. The accumulated data, from the current study and several other recent studies, 9,10,31 may lead to an adjusted recommendation for shorter tourniquet application time but should be interpreted with the knowledge that the study protocols in the current and previous studies were not identical regarding dose of amikacin sulphate and perfusate volume. 
Table 1 Amikacin sulphate concentrations in radiocarpal joint synovial fluid from 5 to 30 minutes after cephalic intravenous regional limb perfusion with $2 \mathrm{~g}$ of amikacin sulphate in 6 horses.

\begin{tabular}{|c|c|c|c|c|c|c|c|c|}
\hline \multicolumn{9}{|c|}{ Synovial fluid amikacin sulphate concentration over time } \\
\hline $\begin{array}{l}\text { Time } \\
\text { (min) }\end{array}$ & $\begin{array}{l}\text { Horse A } \\
310 \mathrm{~kg} \\
\mu \mathrm{g} / \mathrm{mL}\end{array}$ & $\begin{array}{l}\text { Horse B } \\
327 \mathrm{~kg} \\
\mu \mathrm{g} / \mathrm{mL}\end{array}$ & $\begin{array}{l}\text { Horse C } \\
470 \mathrm{~kg} \\
\mu \mathrm{g} / \mathrm{mL}\end{array}$ & $\begin{array}{l}\text { Horse D } \\
285 \mathrm{~kg} \\
\mu \mathrm{g} / \mathrm{mL}\end{array}$ & $\begin{array}{l}\text { Horse E } \\
466 \mathrm{~kg} \\
\mu \mathrm{g} / \mathrm{mL}\end{array}$ & $\begin{array}{l}\text { Horse F } \\
376 \mathrm{~kg} \\
\mu \mathrm{g} / \mathrm{mL}\end{array}$ & $\begin{array}{l}\text { Mean } \\
372 \mathrm{~kg} \\
\mu \mathrm{g} / \mathrm{mL}\end{array}$ & $\begin{array}{l}\text { Standard } \\
\text { deviation }\end{array}$ \\
\hline 5 & 185 & 115 & 337 & 437 & 807 & 175 & 343 & 256.6 \\
\hline 10 & 442 & 479 & 849 & 561 & 1950 & 630 & 818 & 572.7 \\
\hline 15 & 961 & 588 & 1010 & 616 & 1490 & 495 & 860 & 373.2 \\
\hline 20 & 746 & 539 & 1110 & 1680 & 1720 & 343 & 1023 & 582.8 \\
\hline 25 & 918 & 278 & 1040 & 680 & 1380 & 399 & 783 & 413.2 \\
\hline 30 & 753 & 307 & 783 & 386 & 1030 & 534 & 632 & 272.6 \\
\hline
\end{tabular}

Note: Maximum concentration ( $\mathrm{Cmax}$ ) in each horse is indicated with bold numbers. Standard deviations are given to the mean $\mathrm{Cmax}$ at each time point. Values are given in $\mu \mathrm{g} / \mathrm{mL}$.

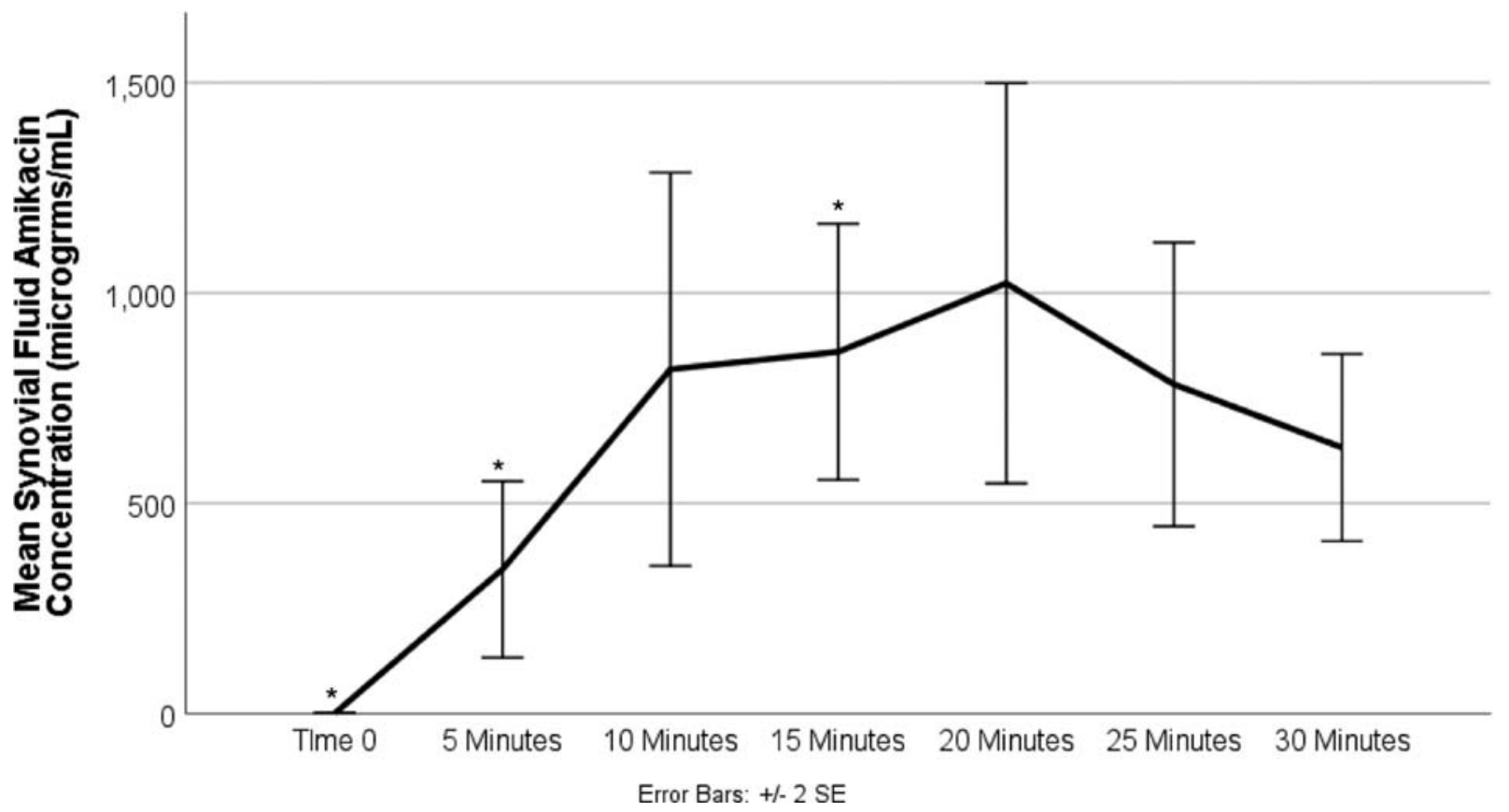

Fig. 1 Mean synovial fluid amikacin sulphate concentration at each time point with standard error bars. The mean synovial fluid amikacin sulphate concentration increased until 20 minutes and, subsequently decreased until 30 minutes. Results of repeated measures analysis of variance pair wise comparisons default (Least Significant Difference test) showed significant differences between time points 0 and 5 minutes and all other time points marked with "*" in the graph. $p$-Values for comparing time point 0 and other time points ranged between $p=0.002$ and 0.022. $p$-Values for comparing time point 5 and other time points were between $p=0.003$ and 0.022 . A significant difference between time point 15 minutes and 30 minutes is marked in the graph with "*." p-Value for the difference between 15 and 30 minutes was $p=0.018$.

The findings of the current study differ from previous publications that found no difference in synovial fluid amikacin sulphate concentrations between the 30-minute time point and earlier sampling time points. ${ }^{10,29}$ We found a significant reduction in the mean amikacin sulphate synovial fluid concentration between the 15- and 30-minute time point and none of the included horses had a $\mathrm{T}_{\max }$ greater than 20 minutes. Although the highest mean synovial fluid concentration was observed at the 20-minute time point, it was not significantly different to the 30-minute time point. This is most likely due to the large standard error accompanying the mean along with the small sample size (-Fig. $\mathbf{1}$ ).
Repeated sedation is commonly needed to limit discomfort and movement throughout the typically used 30 minutes tourniquet application time when performing IVRLP. ${ }^{10} \mathrm{~A}$ shorter tourniquet application time results in a decreased amount of sedation required for the procedure. In horses receiving daily IVRLP, the accompanying sedation could increase the risk of colic since detomidine hydrochloride in combination with butorphanol tartrate significantly delays gastric emptying rate. ${ }^{23,24}$

In addition, the risk of tourniquet side effects would potentially be decreased. Tourniquet side effects have not been investigated in horses but are well known in human medicine as post-tourniquet syndrome. Pain from neural ischaemia and 
complications in wound healing due to wound hypoxia are complications associated with reperfusion injury following prolonged tourniquet application ( $>45-60$ minutes). ${ }^{25,26}$ The risk of these complications could all be decreased with a shorter tourniquet application time. However, the clinical significance of shortening the time from 30 minutes to 20 is speculative and both time points are shorter than the maximal recommended tourniquet duration of 1 hour. ${ }^{25,26}$

In the present study, and in several previous IVRLP studies evaluating synovial fluid amikacin sulphate concentrations in various joints in standing horses, synovial fluid concentration varied widely among horses. ${ }^{8,13,15,32}$ The large variability of antimicrobial synovial fluid concentrations in IVRLP has been suggested to occur due to multiple reasons including tourniquet failure and drug leakage to the systemic circulation caused by limb movement or faulty application of the tourniquet. ${ }^{8,13,15,32}$ The significance of the variability in synovial concentrations, is questionable in the current study since concentrations achieved, did not indicate tourniquet failure and reached above the MIC values of susceptible pathogens most commonly isolated from orthopaedic infections. ${ }^{17,18}$ Reported MIC of amikacin sulphate of commonly isolated pathogens from musculoskeletal infections ranges between 16 and $64 \mu \mathrm{g} / \mathrm{mL} .{ }^{20}$ Although some strains of methicillinresistant Staphylococcus aureus have a reported MIC of 500 $\mu \mathrm{g} / \mathrm{mL}$ the median MIC for amikacin sulphate for methicillinresistant-Staphylococcus aureus in one study was reported to be $32 \mu \mathrm{g} / \mathrm{mL} .{ }^{19}$ Hence, the recommended $C_{\text {max }}$ :MIC ratio of $8: 1$ was reached in all horses but a 10:1 ratio for the most resistant pathogens $(>64 \mu \mathrm{g} / \mathrm{mL}$ ) was only reached in $4 / 6$ horses advocating for a higher dosage in horses suffering from resistant infections. The reported dosage of amikacin sulphate in IVRLP ranges between $500 \mathrm{mg}$ and $3 \mathrm{~g} \cdot{ }^{6-8,10,12-16,20}$ We choose to use $2 \mathrm{~g}$ in this study based on our clinical experience and previous studies that reached desirable MIC levels of synovial fluid amikacin sulphate with this dose. ${ }^{6,16}$ To avoid potential cytotoxicity of amikacin sulphate described after intra-articular administration, the dose could be titrated according to MIC of the cultured pathogens, keeping in mind that bacteriology samples from equine orthopaedic infections frequently yield negative growth. ${ }^{17}$

In the current and previous studies sampling synovial fluid from the carpal joints, the forelimb was flexed during the arthrocentesis. This movement does not appear to influence the synovial fluid amikacin sulphate concentration. $8,10,13,15,20,29,33,34$ The influence of repeated sampling of synovial fluid on the amikacin sulphate synovial fluid concentration is unknown, not accounted for in previous studies, and should be further investigated. The two horses with suspected perivascular leakage had similar synovial fluid concentrations to the other horses, and the swelling disappeared shortly after tourniquet removal. Therefore, the leakage did not interfere with reaching therapeutic amikacin sulphate synovial fluid concentrations.

Peri-neural anaesthesia of the ulnar, median and musculocutaneous nerves was performed to minimize pain caused by the tourniquet. This is suggested to be the most efficient anaesthetic method in IVRLP. ${ }^{8}$ Several studies have evaluated the IVRLP efficacy under general anaesthesia to control for the influence of movement on perfusion. ${ }^{8-10,19}$ A study comparing synovial fluid amikacin sulphate concentrations between anaesthetized and sedated horses, in the radiocarpal joint and metacarpophalangeal joint, did not find significant difference between the two methods. Actually, in that study, general anaesthesia had the effect of decreasing metacarpophalangeal joint synovial fluid concentrations of amikacin sulphate. ${ }^{10}$ Another study concluded that their results did not support the use of general anaesthesia in IVRLP as it had no positive effect on the pharmacokinetics of amikacin sulphate in synovial fluid following IVRLP compared with standing horses and failed to be a clinically relevant solution. ${ }^{8}$

An Esmarch's wide rubber tourniquet was used in this study. Although with the pneumatic tourniquet the pressure is measurable and more consistent, ${ }^{14}$ the wide Esmarch's tourniquet is effective in sustaining haemostasis of the distal limb and allowing accumulation of high concentration of antibiotic medications. ${ }^{14,16,32}$

The high concentration of synovial fluid amikacin sulphate reached in this study is consistent with the findings of other recent studies from our laboratory. This is likely supportive of the technique we are implementing, including the use of the cephalic vein, a wide Esmarch's tourniquet, $2 \mathrm{~g}$ of amikacin sulphate in $100 \mathrm{~mL}$ volume; divided in five $20 \mathrm{~mL}$ syringes, and peripheral nerve anaesthesia. The accuracy and precision of the analysis of the synovial fluid amikacin concentration were $\pm 15 \%$, except for $\pm 20 \%$ in lower level of quantification. These values are in agreement with recommended acceptance criteria (guidance for industry, Food and Drug Administration [FDA]). ${ }^{35}$

There are several limitations to this study, including a small study group with large variation in the results. The same dose of antibiotic medication and perfusate volume was used throughout the study group despite variations in body weight. This uniform dosage could have affected the results, although no significant correlation was found between body weight and synovial fluid amikacin sulphate concentration. Previous studies administering precise dosages did not avoid large variations in synovial fluid antimicrobial concentrations through individual dosages. ${ }^{13}$ Additionally, since only radiocarpal joint synovial fluid was sampled, these results cannot be applied to other synovial structures, subcutaneous tissue, skin or bone. At last, although the study protocol was completely uniform, four different veterinary surgeons applied the tourniquet, performed the IVRLP and sampled the radiocarpal joint.

The results of the present study support the reduction in tourniquet application time from 30 to 20 minutes for cephalic vein IVRLP with $2 \mathrm{~g}$ of amikacin sulphate in $100 \mathrm{~mL}$ volume when targeting the radiocarpal joint. However, further investigations are required to evaluate if this is applicable to perfusion of other tissues and other synovial structures and to ensure the clinical validity of this protocol.

\section{Authors' Contributions}

Kajsa Gustafsson, Amos J. Tatz, Roee Dahan, and Gal Kelmer contributed to the conception of study. Kajsa Gustafsson, Amos J. Tatz, Roee Dahan, Malka Britzi, and 
Gal Kelmer contributed to acquisition of data. Kajsa Gustafsson, Amos J. Tatz, Roee Dahan, Malka Britzi, Stefan Soback, Gila A. Sutton, and Gal Kelmer contributed to data analysis and interpretation. All authors drafted, revised and approved the submitted manuscript.

\section{Conflict of Interest}

None declared.

\section{References}

1 Kelmer G. Regional limb perfusion in horses. Vet Rec 2016;178 (23):581-584

2 Smith LJ, Marr CM, Payne RJ, Stoneham SJ, Reid SW. What is the likelihood that Thoroughbred foals treated for septic arthritis will race? Equine Vet J 2004;36(05):452-456

3 Richardson DW, Ahern BJ. Synovial and osseous infections. In: Auer J, Stick JA, eds. Equine Surgery. 4th ed. St. Louis, MO: Elsevier; 2012:1189-1200

4 Lescun TB, Ward MP, Adams SB. Gentamicin concentrations in synovial fluid and joint tissues during intravenous administration or continuous intra-articular infusion of the tarsocrural joint of clinically normal horses. Am J Vet Res 2006;67(03):409-416

5 Båverud V, Gustafsson A, Franklin A, Aspán A, Gunnarsson A. Clostridium difficile: prevalence in horses and environment, and antimicrobial susceptibility. Equine Vet J 2003;35(05):465-471

6 Kelmer G, Bell GC, Martin-Jimenez T, et al. Evaluation of regional limb perfusion with amikacin using the saphenous, cephalic, and palmar digital veins in standing horses. J Vet Pharmacol Ther 2013;36(03):236-240

7 Scheuch BC, Van Hoogmoed LM, Wilson WD, et al. Comparison of intraosseous or intravenous infusion for delivery of amikacin sulfate to the tibiotarsal joint of horses. Am J Vet Res 2002;63(03):374-380

8 Mahne AT, Rioja E, Marais HJ, Villarino NF, Rubio-Martinez LM. Clinical and pharmacokinetic effects of regional or general anaesthesia on intravenous regional limb perfusion with amikacin in horses. Equine Vet J 2014;46(03):375-379

9 Murphey ED, Santschi EM, Papich MG. Regional intravenous perfusion of the distal limb of horses with amikacin sulfate. J Vet Pharmacol Ther 1999;22(01):68-71

10 Aristizabal FA, Nieto JE, Guedes AG, et al. Comparison of two tourniquet application times for regional intravenous limb perfusions with amikacin in sedated or anesthetized horses. Vet J 2016;208:50-54

11 Kelmer G, Tatz A, Bdolah-Abram T. Indwelling cephalic or saphenous vein catheter use for regional limb perfusion in 44 horses with synovial injury involving the distal aspect of the limb. Vet Surg 2012;41(08):938-943

12 Alkabes SB, Adams SB, Moore GE, Alkabes KC. Comparison of two tourniquets and determination of amikacin sulfate concentrations after metacarpophalangeal joint lavage performed simultaneously with intravenous regional limb perfusion in horses. Am J Vet Res 2011;72(05):613-619

13 Beccar-Varela AM, Epstein KL, White CL. Effect of experimentally induced synovitis on amikacin concentrations after intravenous regional limb perfusion. Vet Surg 2011;40(07):891-897

14 Levine DG, Epstein KL, Ahern BJ, Richardson DW. Efficacy of three tourniquet types for intravenous antimicrobial regional limb perfusion in standing horses. Vet Surg 2010;39(08): 1021-1024

15 Zantingh AJ, Schwark WS, Fubini SL, Watts AE. Accumulation of amikacin in synovial fluid after regional limb perfusion of amikacin sulfate alone and in combination with ticarcillin/clavulanate in horses. Vet Surg 2014;43(03):282-288

16 Oreff GL, Dahan R, Tatz AJ, Raz T, Britzi M, Kelmer G. The effect of perfusate volume on amikacin concentration in the metacarpophalangeal joint following cephalic regional limb perfusion in standing horses. Vet Surg 2016;45(05):625-630
17 Hepworth-Warren KL, Wong DM, Fulkerson CV, Wang C, Sun Y. Bacterial isolates, antimicrobial susceptibility patterns, and factors associated with infection and outcome in foals with septic arthritis: 83 cases (1998-2013). J Am Vet Med Assoc 2015;246(07):785-793

18 Moore RM, Schneider RK, Kowalski J, Bramlage LR, Mecklenburg LM, Kohn CW. Antimicrobial susceptibility of bacterial isolates from 233 horses with musculoskeletal infection during 19791989. Equine Vet J 1992;24(06):450-456

19 Caron JP, Bolin CA, Hauptman JG, Johnston KA. Minimum inhibitory concentration and postantibiotic effect of amikacin for equine isolates of methicillin-resistant Staphylococcus aureus in vitro. Vet Surg 2009;38(05):664-669

20 Harvey A, Kilcoyne I, Byrne BA, Nieto J. Effect of dose on intraarticular amikacin sulfate concentrations following intravenous regional limb perfusion in horses. Vet Surg 2016;45(08):1077-1082

21 Pezzanite L, Chow L, Soontararak S, Phillips J, Goodrich L, Dow S. Amikacin induces rapid dose-dependent apoptotic cell death in equine chondrocytes and synovial cells in vitro. Equine Vet J 2020; 00:1-10

22 Gleerup KB, Forkman B, Lindegaard C, Andersen PH. An equine pain face. Vet Anaesth Analg 2015;42(01):103-114

23 Doherty TJ, Andrews FM, Provenza MK, Frazier DL. The effect of sedation on gastric emptying of a liquid marker in ponies. Vet Surg 1999;28(05):375-379

24 Sutton DG, Preston T, Christley RM, Cohen ND, Love S, Roussel AJ. The effects of xylazine, detomidine, acepromazine and butorphanol on equine solid phase gastric emptying rate. Equine Vet $\mathrm{J}$ 2002;34(05):486-492

25 Estebe JP, Davies JM, Richebe P. The pneumatic tourniquet: mechanical, ischaemia-reperfusion and systemic effects. Eur J Anaesthesiol 2011;28(06):404-411

26 Oragui E, Parsons A, White T, Longo UG, Khan WS. Tourniquet use in upper limb surgery. Hand (N Y) 2011;6(02):165-173

27 Singh AP, Singh J, Peshin PK, Nigam JM, Chawla SK. Effects of limb tourniquet ischemia on local and systemic acid-base and blood gases of cattle. Can J Comp Med 1982;46(04):405-409

28 Richardson DW, Stewart S. Synovial and osseous infections. In: Auer J, Stick JA, eds. Equine Surgery. 5th ed. St. Louis, MO: Elsevier; 2019:1458-1470

29 Kilcoyne I, Dechant JE, Nieto JE. Evaluation of 10-minute versus 30-minute tourniquet time for intravenous regional limb perfusion with amikacin sulfate in standing sedated horses. Vet Rec 2016;178(23):585-585

30 Moyer W, Schumacher J, Schumacher J. A guide to equine joint injection and regional anesthesia. Yardley, PA, Veterinary Learning Systems; 2007

31 Kilcoyne I, Nieto JE, Knych HK, Dechant JE. Time required to achieve maximum concentration of amikacin in synovial fluid of the distal interphalangeal joint after intravenous regional limb perfusion in horses. Am J Vet Res 2018;79(03):282-286

32 Oreff GL, Tatz AJ, Dahan R, et al. Pharmacokinetics of ceftazidime after regional limb perfusion in standing horses. Vet Surg 2017;46 (08):1120-1125

33 Schoonover MJ, Moser DK, Young JM, Payton ME, Holbrook TC. Effects of tourniquet number and exsanguination on amikacin concentrations in the radiocarpal and distal interphalangeal joints after low volume intravenous regional limb perfusion in horses. Vet Surg 2017;46(05):675-682

34 Moser DK, Schoonover MJ, Holbrook TC, Payton ME. Effect of regional intravenous limb perfusate volume on synovial fluid concentration of amikacin and local venous blood pressure in the horse. Vet Surg 2016;45(07):851-858

35 Food and Drug Administration. Guidance for Industry: Bioanalytical Method Validation. US Department of Health and Human Services, FDA, Center for Drug Evaluation and Research; Rockville, MD: 2018. Available at: http://www.fda.gov/downloads/Drugs/ GuidanceComplianceRegulatoryInformation/Guidances/ UCM070107.pdf 